\title{
Visual outcomes comparing surgical techniques for management of severe idiopathic intracranial hypertension
}

\author{
Steven E. Feldon, M.D., M.B.A. \\ Departments of Ophthalmology, Neurology, Neurological Surgery, and Vision Science, University of \\ Rochester School of Medicine and Dentistry, and University of Rochester Eye Institute, \\ Rochester, New York
}

\begin{abstract}
Object. The object of this study was to compare surgical techniques for management of visual loss in idiopathic intracranial hypertension (IIH) unresponsive to medical treatment.

Methods. The published literature was reviewed using electronic and manual search techniques. Articles were categorized based upon the surgical procedure performed, including optic nerve sheath decompression (ONSD), also called optic nerve sheath fenestration; intracranial venous sinus stent placement; ventriculoperitoneal (VP) shunt placement; and lumboperitoneal (LP) shunt placement. Demographic data and visual outcome of the cumulative experience were compiled and compared for each type of surgical intervention.

Results. Seventeen patients treated by stent placement, 31 by VP shunt placement, 44 by LP shunt placement, and 252 patients by ONSD were identified in the literature; average follow-up ranged from 11.8 months for patients treated with stents to 57.2 months for those treated with LP shunts. Improved or resolved vision deficit was noted in $38.7 \%$ of patients after VP shunt placement, $47 \%$ of patients after stent placement, $44.6 \%$ of patients after LP shunt placement, and $80 \%$ of eyes after ONSD. Visual worsening was rare for all procedures evaluated.

Conclusions. There is a paucity of information regarding visual outcomes from intracranial venous stent placement and cerebrospinal fluid diversion procedures. Visual outcomes from ONSD are better documented and appear to be superior to other surgical techniques for management of IIH. Further studies with improved data collection regarding visual outcomes are needed for surgical procedures other than ONSD in order to assess their possible value as treatments for visual loss in IIH.(DOI: 10.3171/FOC-07/11/E6)
\end{abstract}

\begin{abstract}
KEY WORDS • cerebrospinal fluid shunt • idiopathic intracranial hypertension • intracranial venous stent $\bullet$ optic nerve sheath decompression $\bullet$ visual function
\end{abstract}

I DIOPATHIC intracranial hypertension is a syndrome consisting of elevation in intracranial pressure without evidence of structural lesion or alteration of CSF. Symptoms and signs must be attributable only to increased intracranial pressure or papilledema. ${ }^{15}$ The most common manifestations of the disease are headache and an assortment of visual symptoms, including transient obscurations of vision, diplopia from abducent nerve paresis, and visual loss associated with the effects of papilledema. Pulsatile tinnitus may also be present. Most commonly occurring in obese women, ${ }^{11,35}$ this syndrome has also been called pseudotumor cerebri and benign intracranial hypertension.

As the term IIH connotes, the etiology of this entity is unknown, although several hypotheses exist. An increase in CSF secretion or a decrease in CSF absorption caused by unknown hormonal or proinflammatory mediators has

Abbreviations used in this paper: BMI = Body Mass Index; $\mathrm{CSF}=$ cerebrospinal fluid; $\mathrm{IIH}=$ idiopathic intracranial hypertension; $\mathrm{LP}=$ lumboperitoneal; ONSD = optic nerve sheath decompression; $\mathrm{VP}=$ ventriculoperitoneal. been suggested. Another concept is based upon increased intracranial venous pressure due either to transmission of high intraabdominal pressure or to relative (or functional) stenosis of the intracranial venous sinuses. These concepts are presented and discussed in an excellent review by Friedman. ${ }^{15}$

Multiple therapeutic alternatives are available for management of IIH. Medical therapy is directed toward reducing CSF secretion and increasing CSF absorption with diuretics such as furosamide and carbonic anhydrase inhibitors such as acetazolamide., ${ }^{9,41}$ Weight loss, through diet or surgical intervention, is advocated to reduce intraabdominal pressure or change secretion of offending but unknown mediators. . $^{21,25,40}$ Cerebrospinal fluid diversion procedures address the effects of $\mathrm{IIH}$ rather than purported causes, simply draining excess CSF out of the subarachnoid space. Such procedures include placement of LP shunts, ${ }^{5,12,31}$ VP shunts, ${ }^{6,29}$ and foramen magnum-atrial shunts, ${ }^{22}$ as well as subtemporal decompressions. Optic nerve sheath decompression may work in part through CSF diversion and in part by blocking transmission of intracranial pressure to the optic disc at the level of the lamina 
cribrosa. ${ }^{17,23,42}$ Based upon the concept that there is functional or actual stenosis of the intracranial venous outflow, intravascular stenting of the venous sinuses has been advocated as a yet another mode of treatment. ${ }^{4,10,14,18-20,24,33,34,36}$

Primary indications for the treatment of IIH are intractable headache and vision loss. Unfortunately, there are no evidence-based studies in which the risks and benefits of various interventions are assessed. ${ }^{28}$ Furthermore, the relative rarity of severe complications of IIH and the variability in presentation make prospective clinical trials very problematic. Thus, in the absence of a scientifically demonstrated advantage for any single treatment, the indications for a particular therapy differ widely depending upon the biases of the treating physicians.

Although there may be some advocates of primary surgical therapy, the consensus is that maximum tolerated medical therapy (diuretics and weight loss) should be tried before invasive options are considered. ${ }^{15}$ Case studies suggest that ONSD is generally effective for managing visual loss, whereas diversion and perhaps stent placement procedures may be more beneficial for managing headache. One reason for this impression might be that ophthalmologists follow up patients with visual loss and, therefore, are more likely to suggest a procedure performed by ophthalmologists. By similar reasoning, neurologists would then be more likely to see patients with headache and recommend a neurosurgical or neurovascular approach. All interventions are associated with known risks, of which the most likely is a failure to sustain benefit.

The purpose of the present study is to directly compare the published experience of surgical techniques commonly advocated for treatment of visual loss in IIH. This analysis is intended to investigate the visual benefit of LP shunts, VP shunts, and venous sinus stents, relative to ONSD. A further intention is to recommend improvements in data collection that will increase the clinical value of future studies assessing visual outcomes following surgical management of IIH.

\section{Clinical Material and Methods}

Peer-reviewed published articles were retrieved using online MEDLINE searches. Key words used in the primary search included the following: idiopathic intracranial hypertension, pseudotumor cerebri, and benign intracranial hypertension. Subsidiary searches were conducted for lumboperitoneal shunts, ventriculoperitoneal shunts, optic nerve sheath decompression and intracranial venous stents. Web of Science Citation Index searches and reference reviews were conducted on relevant recovered articles. Series or cases that did not include vision data were not considered. Excluded from analysis of ONSD were individual case reports, series of less than 10 cases, topic reviews, and articles not written in English. One series was excluded because publication dates suggested overlapping cohorts. ${ }^{38}$

Because of a paucity of reports, individual cases and small series in the nonpediatric age group were included if they were related to stents and CSF diversion techniques and if there were vision data available. Articles providing summary data only (no individual case data given) were excluded. Cases involving venous sinus occlusion or other secondary causes of increased intracranial pressure within an included series were excluded from the analysis.

For ONSD, summary data were combined to develop an aggregate of experience with the procedure. For all other surgical interventions, data available for the individual cases in each series were combined in order to assess the cumulative reported experience with each procedure. The authors of one report of a series of cases in which ONSD was performed subcategorized cases as acute and chronic. ${ }^{39}$ The data for chronic IIH were evaluated separately from data pertaining to acute cases.

For all studies in which multiple time points were reported for visual outcome, the 6-month data were utilized. Criteria for visual improvement or deterioration were based upon the investigators' definitions. If no definition was provided, any reported change was included. Transient obscurations of vision and enlarged blinds spots on visual field testing, when specified, were not considered to constitute vision loss.

\section{Results}

\section{Optic Nerve Sheath Decompression}

Seven retrospective series were identified which met the inclusion criteria for the study. 1,3,7,8,16,37,39 In all, 423 eyes of 252 patients were evaluated with a mean follow-up of 21.1 months (range 0-121 months). The patients' mean age was 33.6 years (range 6-72 years), and $83 \%$ of the patients were female. Of interest, the majority $(59 \%)$ of surgeries were bilateral. In 90\% (226) of cases, ONSD was the first surgery performed. Visual acuity was improved in 50\% of eyes (113) and visual field was improved in $72 \%$ of eyes (226). Visual acuity or visual field worsened in only 42 cases $(11 \%)$. The reoperation rate was $12 \%$, with repeated ONSD being the most common procedure $(6 \%)$ followed by CSF diversion procedure (4\%). Results are summarized in Table 1. Note that not all data was available for all cases; thus, denominators used in calculating percentages differed.

\section{Intracranial Venous Sinus Stents}

Stent placement was the least frequently reported of all surgical procedures advocated for management of IIH. Only 17 cases, followed up for an average of 11.8 months (range 5-24 months), met the inclusion criteria. ${ }^{18,19,34,36}$ Indications for surgery included both headache and vision problems in 12 cases $(70.6 \%)$, vision problems only in 1 case $(5.9 \%)$, and headache in $4(23.5 \%)$. The average age was 30 years with a range of $15-52$ years. Fifteen $(88.2 \%)$ were women and all were obese, with a mean BMI of 33.9 (the threshold for obesity being an index greater than 25 being defined as obese). In the majority of cases (58.8\%), stent placement was the first procedure performed. Resolution of papilledema occurred in 8 cases $(47.1 \%)$, improvement in papilledema occurred in 1 case $(5.9 \%)$, and there was no change in papilledema in 5 cases $(29.4 \%)$ In 3 cases, information regarding disc swelling was unavailable.

Headaches resolved in 8 cases $(47.1 \%)$ and improved in 2 others $(11.8 \%)$. Headaches persisted without change in 4 cases $(23.5 \%)$, and no information was available regarding headache severity in 1 case. Vision recovered completely in 
Visual outcomes in surgical management of IIH

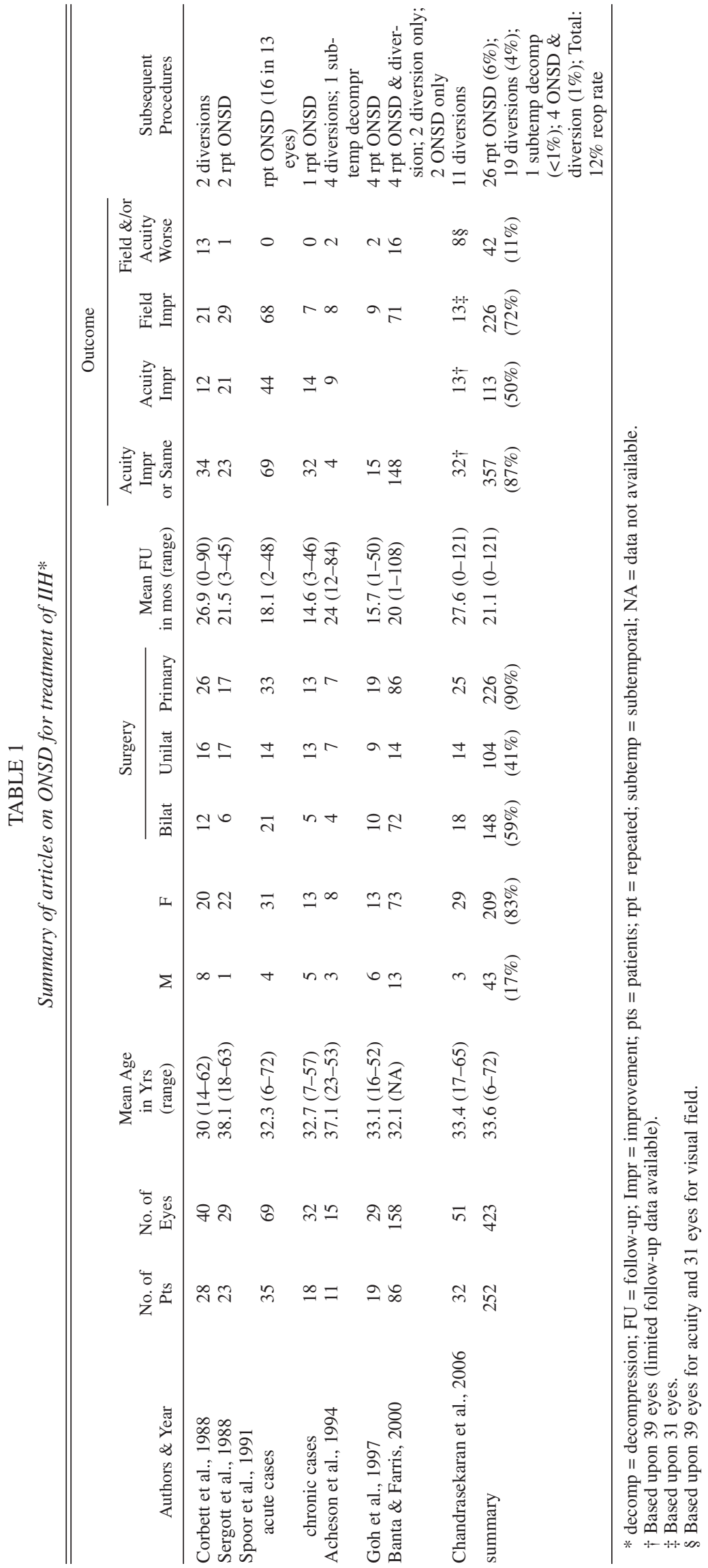


3 cases (17.6\%) and improved in 5 others (29.4\%). There was no change in vision in 4 cases $(23.5 \%)$, and no information regarding vision change was available in 4 others. Results are summarized in Table 2.

\section{Ventriculoperitoneal Shunts}

Reports of 2 case series and 2 single cases were identified in which VP shunts were utilized for treatment of IIH and the criteria for inclusion in the study were met. ${ }^{4,6,29,34}$ The mean age of the 31 patients was 32.4 years (range 6-63 years), and $23(74.2 \%)$ were female. Average followup was 48.3 months (range 1-153.6 months). The mean BMI was 31.1 for the 18 cases for which information was provided. Placement of a VP shunt was the primary surgical procedure in 16 cases (51.6\%). Papilledema resolved in all of the 17 cases for which data pertaining to that condition were reported. Headache resolved in 1 case and improved in another, but information regarding this symptom was not available for 29 patients. Vision was noted to improve in 12 cases $(38.7 \%)$. In the majority of patients, there was no change in vision (19 cases, 61.3\%). Results are summarized in Table 3.

\section{Lumboperitoneal Shunts}

Reports of 2 case series and 2 single cases were identified in which LP shunts were used for treatment of IIH the criteria for inclusion in the study were met. ${ }^{5,12,31,34}$ Average follow-up was 58.7 months (range 0.2-278 months). The 44 patients' mean age was 30.7 years (range 19-52 years), and $92.9 \%$ were women, but in 1 series of 16 cases, the age and sex of individual patients was not reported. ${ }^{5}$ The BMI was provided only in the 2 case reports and was 27 in each. Placement of an LP shunt was identified as the primary surgical intervention in 27 (96.4\%) of 28 cases for which information was provided.

Papilledema resolved in 10 cases $(22.7 \%)$ and improved in another 7 (15.9\%), but information was not available for the majority of patients (27 cases [61.4\%]). Headache resolved in 8 cases (18.2\%) and improved in another 12 $(27.3 \%)$. Again, information was not provided in the majority (24 cases [54.5\%]). Vision symptoms or signs resolved in 7 cases $(15.9 \%)$ and improved in $13(29.5 \%)$. There was no change in vision in 7 cases $(15.9 \%)$ and worsening occurred in $2(4.5 \%)$. Results are summarized in Table 4.

\section{Discussion}

Surgical procedures are commonly utilized for treatment of IIH when medical treatments fail or are poorly tolerated. However, this review demonstrates that when one excludes the data on ONSD (252 cases), there is a paucity of data regarding the effect of various surgical interventions on vision (17-44 cases, depending on the intervention). The most likely explanation for the difference in data collection between ONSD and other interventions is that ONSD is performed by ophthalmologists who routinely monitor visual function, whereas the other surgeries are performed by neurosurgeons or neurovascular interventionalists, who depend upon others for visual assessment. In studies other than those on ONSD, either the visual function of patients may not be readily available to the investigators or visual outcome may not be the primary thrust of the study.

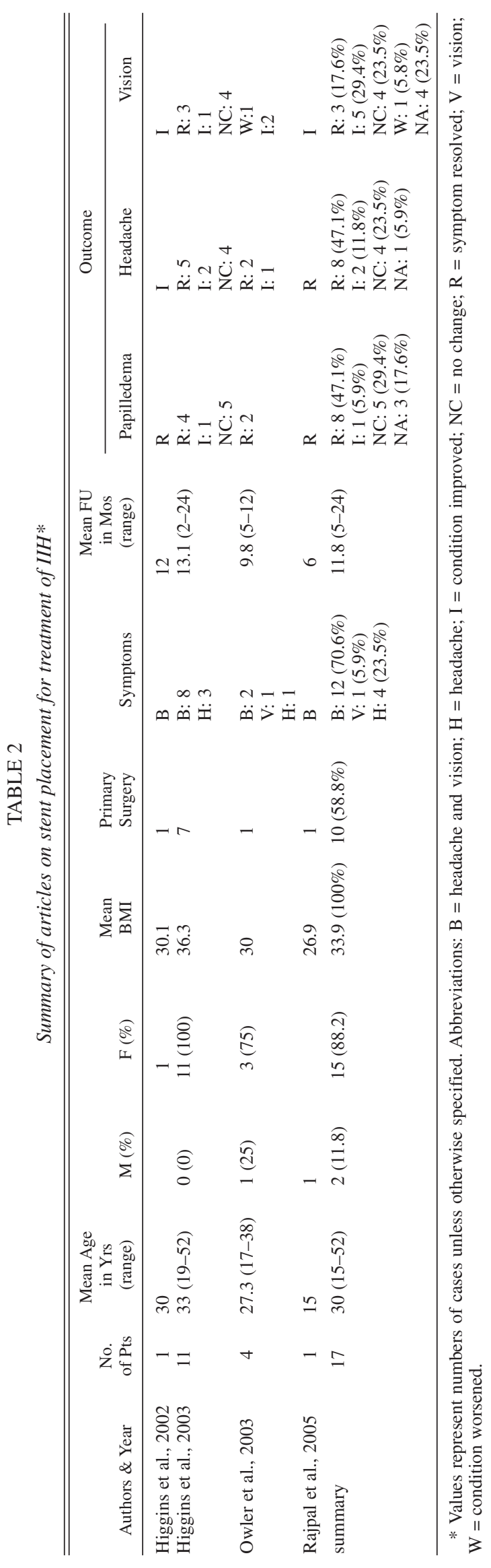

Neurosurg. Focus / Volume 23 / November, 2007 


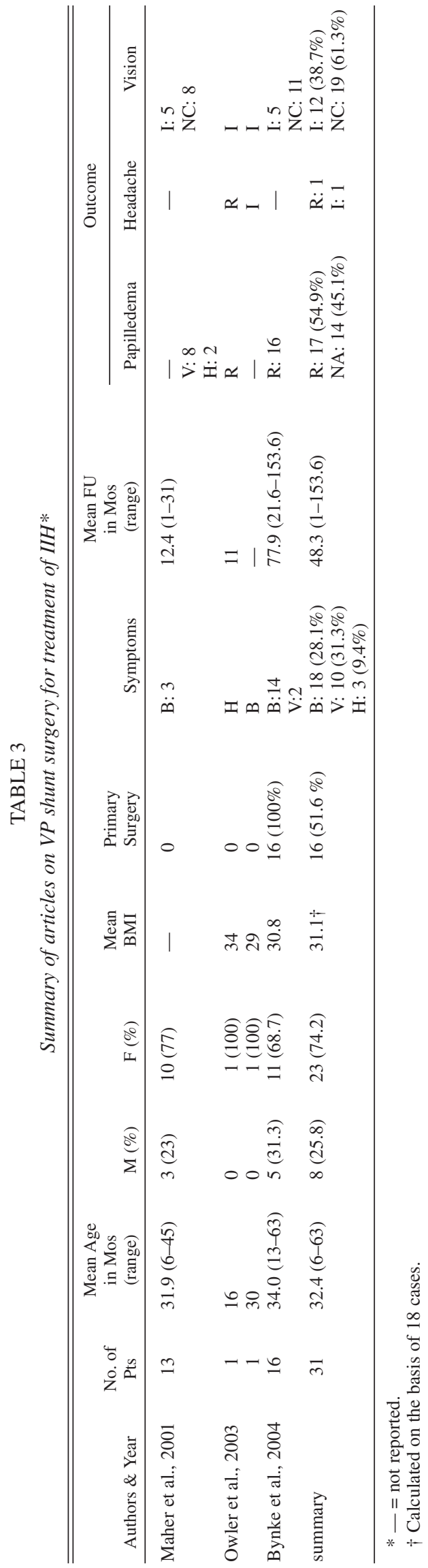

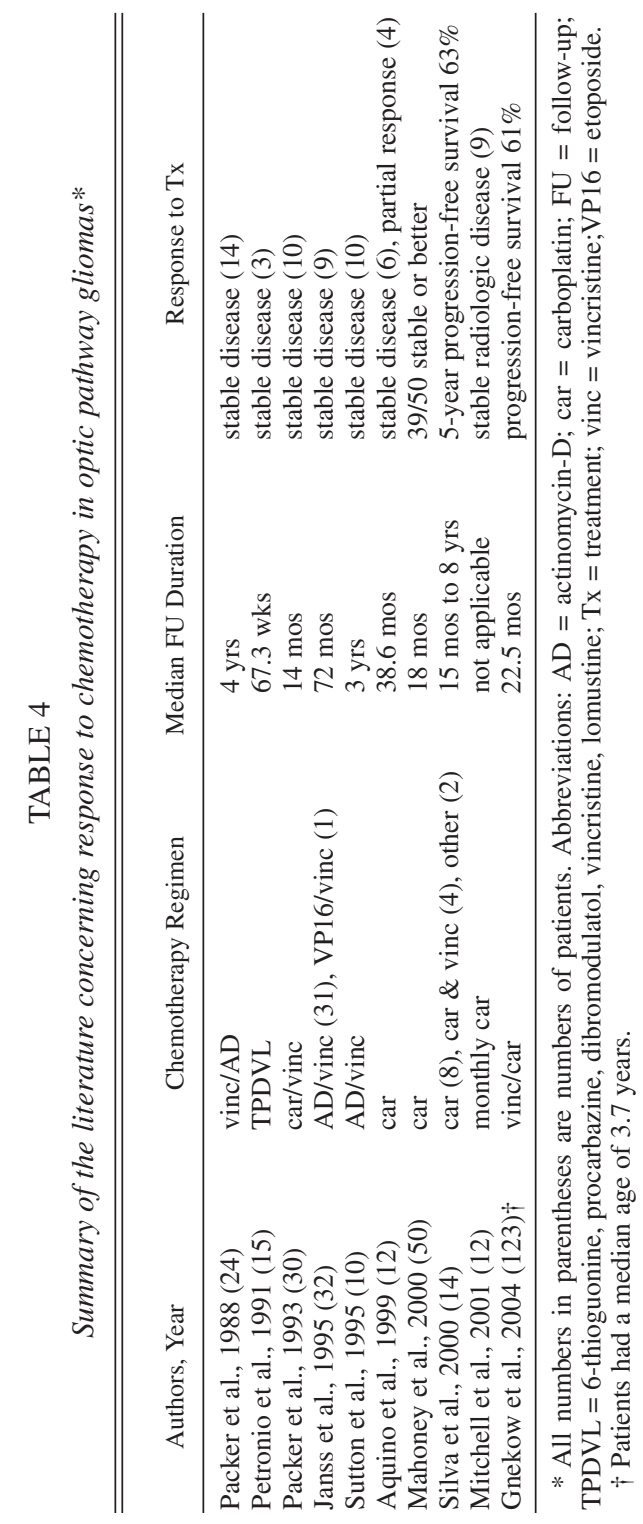


TABLE 5

Summary of studies comparing visual outcomes following surgical treatment of IIH

\begin{tabular}{|c|c|c|c|c|c|c|c|c|}
\hline Procedure & $\begin{array}{l}\text { No. } \\
\text { of } \\
\text { Pts }\end{array}$ & $\begin{array}{c}\text { Mean Age } \\
\text { in Years } \\
\text { (range) }\end{array}$ & $\mathrm{M}(\%)$ & $\mathrm{F}(\%)$ & $\begin{array}{c}\text { Mean } \\
\text { FU in } \\
\text { Mos (range) }\end{array}$ & $\begin{array}{c}\text { Primary } \\
\text { Surgery } \\
(\%)\end{array}$ & $\begin{array}{l}\text { Vision } \\
\text { Impr } \\
(\%)\end{array}$ & $\begin{array}{c}\text { Vision } \\
\text { Worse } \\
(\%)\end{array}$ \\
\hline stent placement & 17 & $30(15-52)$ & $2(12)$ & $15(88)$ & $11.8(2-26)$ & $10(58.8)$ & $8(47.0)$ & $1(5.8)$ \\
\hline VP shunt placement & 31 & $32.4(6-63)$ & $8(26)$ & $24(74)$ & $47.3(1-154)$ & $16(52)$ & $12(38.7)$ & 0 \\
\hline LP shunt placement & 44 & $30.7(19-52)$ & $2\left(4.5^{*}\right)$ & $26(59 *)$ & $57.2(1-278)$ & $26(57)$ & $20(44.6)$ & $2(4.5)$ \\
\hline ONSD & 252 & $33.6(6-72)$ & $43(17)$ & $209(83)$ & $21.1(0-121)$ & $226(90)$ & 339 eyes (80) & 0 \\
\hline ONSD in chronic cases & 18 & $32.7(7-57)$ & $5(28)$ & $13(72)$ & $14.6(3-46)$ & $13(72)$ & 21 eyes (66) & 0 \\
\hline
\end{tabular}

* Sex not specified in $36.5 \%$ of cases.

Difficulties in interpreting the results of this study are numerous and include the following: retrospective case series and case reports, small sample sizes, incomplete and variable data collection, differing definitions for meaningful change in outcome, differing indications for surgery, varying duration of postoperative assessment. Despite the considerable limitations of the present analysis, direct comparisons of differing surgical techniques may provide some insights into patient selection as well as beneficial or deleterious effects of treatment. All the case series report on patients with remarkably similar demographics, with a high percentage of women (59-88\%) of similar age (average range 30.4-33.6). Although outcome data was reported at 6 months as a rule, the mean duration of follow-up was much longer for VP and LP shunt studies (47.3 and 57.2 months, respectively) compared to studies of stent placement (11.8 month) and ONSD (21.1 months) (Table 5). Shunt placement procedures may be more prone to failure or in other ways have more complicated courses, perhaps explaining the longer follow-up periods. The use of stents for treatment of IIH is relatively new, so follow-up is expected to be of shorter duration.

Optic nerve sheath decompression seems to have been a primary procedure in nearly all cases (90\%) in which it was performed, whereas placement of a shunt or stents was as a primary procedure in just over half the cases in which it was performed (range 52-58.8\% of cases). Although there are instances of poor visual outcome following surgical procedures for treatment of $\mathrm{IIH}, 2,13,26,27,30,32$ in the articles used for the current analysis remarkably little postoperative visual loss was reported (mean incidence of $0-12 \%$ for the various procedures). Visual improvement or resolution was noted much more frequently in the ONSD group (80\% of eyes) compared to the other groups (38.7-47\% of patients). This remarkable difference may have several explanations. One possibility is that these latter interventions are performed in more chronic cases, less amenable to improvement. Addressing this point, Spoor et al. ${ }^{39}$ analyzed their chronic cases separately. Although the percentage of visual improvement is lower for patients with chronic IIH compared with the overall cohort (66\% compared with $80 \%$ improvement), chronic disease is probably not the full explanation for differences in visual outcome between ONSD and other procedures. Another possibility is selection bias toward headache rather than visual loss as the primary indication for neurosurgical interventions; conversely, there may be selection bias toward visual loss rather than headache as the primary indication for ONSD. Furthermore, better visual assessment for patients in the ONSD series may have detected more sub- tle visual improvement in these cases relative to cases in which patients underwent other interventions. Finally, 6 months may be too short a follow-up period to assess the true benefit of ONSD. Spoor et al..$^{38}$ have shown that deterioration of visual field may be identified in 35\% of patients assessed at 3-5 years after undergoing ONSD.

\section{Conclusions}

One important conclusion from the current analysis is that better data are needed if a rational management plan is to emerge for the surgical treatment of IIH. Although there is always a clamor for randomized, prospective clinical trials to assess visual outcomes following surgery, practical issues including sample size and expense make an evidence-based approach unlikely. In the absence of a prospective clinical trial, more case series may provide data that will be helpful to clinical decision-making. Such series should include careful preoperative and postoperative documentation of visual function and optic disc appearance. Recommended functional measures include best corrected visual acuity, color vision, and computerized visual fields. Despite many caveats, in the absence of a more definitive study, the current analysis suggests that ONSD is the preferred primary surgical technique for managing the visual symptoms and signs of IIH resistant to medical therapy.

\section{References}

1. Acheson JF, Green WT, Sanders MD: Optic nerve sheath decompression for the treatment of visual failure in chronic raised intracranial pressure. J Neurol Neurosurg Psychiatry 57: 1426-1429, 1994

2. Alleyne CH Jr, Shutter LA, Colohan ART: Cranial migration of a lumboperitoneal shunt catheter. South Med J 89:634-636, 1996

3. Banta JT, Farris BK: Pseudotumor cerebri and optic nerve sheath decompression. Ophthalmology 107:1907-1912, 2000

4. Bono F, Giliberto C, Mastrandrea C, Cristiano D, Lavano A, Fera $\mathrm{F}$, et al: Transverse sinus stenoses persist after normalization of the CSF pressure in IIH. Neurology 65:1090-1093, 2005

5. Burgett RA, Purvin VA, Kawasaki A: Lumboperitoneal shunting for pseudotumor cerebri. Neurology 49:734-739, 1997

6. Bynke G, Zemack G, Bynke H, Romner B: Ventriculoperitoneal shunting for idiopathic intracranial hypertension. Neurology 63:1314-1316, 2004

7. Chandrasekaran S, McCluskey P, Minassian D, Assaad N: Visual outcomes for optic nerve sheath fenestration in pseudotumor cerebri and related conditions. Clin Experiment Ophthalmol 34:661-665, 2006

8. Corbett JJ, Nerad JA, Tse DT, Anderson RL: Results of optic 
nerve sheath fenestration for pseudotumor cerebri. The lateral orbitotomy approach. Arch Ophthalmol 106:1391-1397, 1988

9. Corbett JJ, Thompson HS: The rational management of idiopathic intracranial hypertension. Arch Neurol 46:1049-1051, 1989

10. De Simone R, Marano E, Fiorillo C, Briganti F, Di Salle F, Volpe A, et al: Sudden re-opening of collapsed transverse sinuses and longstanding clinical remission after a single lumbar puncture in a case of idiopathic intracranial hypertension. Pathogenetic implications. Neurol Sci 25:342-344, 2005

11. Durcan FJ, Corbett JJ, Wall M: The incidence of pseudotumor cerebri. Population studies in Iowa and Louisiana. Arch Neurol 45:875-877, 1988

12. Eggenberger ER, Miller NR, Vitale S: Lumboperitoneal shunt for the treatment of pseudotumor cerebri. Neurology 46:1524-1530, 1996

13. Flynn WJ, Westfall CT, Weisman JS: Transient blindness after optic nerve sheath fenestration. Am J Ophthalmol 117:678-679, 1994

14. Friedman DI: Cerebral venous pressure, intra-abdominal pressure, and dural venous sinus stenting in idiopathic intracranial hypertension. J Neuroophthalmol 26:61-64, 2006

15. Friedman DI: Pseudotumor cerebri. Neurol Clin 22:99-131, vi, 2004

16. Goh KY, Schatz NJ, Glaser JS: Optic nerve sheath fenestration for pseudotumor cerebri. J Neuroophthalmol 17:86-91, 1997

17. Hamed LM, Tse DT, Glaser JS, Byrne SF, Schatz NJ: Neuroimaging of the optic nerve after fenestration for management of pseudotumor cerebri. Arch Ophthalmol 110:636-639, 1992

18. Higgins JN, Cousins C, Owler BK, Sarkies N, Pickard JD: Idiopathic intracranial hypertension: 12 cases treated by venous sinus stenting. J Neurol Neurosurg Psychiatry 74:1662-1666, 2003

19. Higgins JN, Owler BK, Cousins C, Pickard JD: Venous sinus stenting for refractory benign intracranial hypertension. Lancet 359:228-230, 2002

20. Higgins JN, Tipper G, Varley M, Pickard JD: Transverse sinus stenoses in benign intracranial hypertension demonstrated on CT venography. Br J Neurosurg 19:137-140, 2005

21. Johnson LN, Krohel GB, Madsen RW, March GA Jr: The role of weight loss and acetazolamide in the treatment of idiopathic intracranial hypertension (pseudotumor cerebri). Ophthalmology 105:2313-2317, 1998

22. Johnston IH, Sheridan MM: CSF shunting from the cisterna magna: a report of 16 cases. Br J Neurosurg 7:39-43, 1993

23. Keltner JL: Optic nerve sheath decompression. How does it work? Has its time come? Arch Ophthalmol 106:1365-1369, 1988

24. Kollar C, Parker G, Johnston I: Endovascular treatment of cranial venous sinus obstruction resulting in pseudotumor syndrome. Report of three cases. J Neurosurg 94:646-651, 2001

25. Kupersmith MJ, Gamell L, Turbin R, Peck V, Spiegel P, Wall M: Effects of weight loss on the course of idiopathic intracranial hypertension in women. Neurology 50:1094-1098, 1998

26. Lee AG: Visual loss as the manifesting symptom of ventriculoperitoneal shunt malfunction. Am J Ophthalmol 122:127-129, 1996

27. Liu GT, Volpe NJ, Schatz NJ, Galetta SL, Farrar JT, Raps EC: Severe sudden visual loss caused by pseudotumor cerebri and lumboperitoneal shunt failure. Am J Ophthalmol 122:129-131, 1996

28. Lueck C, Mcllwaine G: Interventions for idiopathic intracranial hypertension. Cochrane Database Syst Rev 3:CD003434, 2002

29. Maher CO, Garrity JA, Meyer FB: Refractory idiopathic intracranial hypertension treated with stereotactically planned ventriculoperitoneal shunt placement. Neurosurg Focus 10(2):E1, 2001

30. Mauriello JA Jr, Shaderowfsky P, Gizzi M, Frohman L: Management of visual loss after optic nerve sheath decompression in patients with pseudotumor cerebri. Ophthalmology 102: 441-445, 1995

31. McGonigal A, Bone I, Teasdale E: Resolution of transverse sinus stenosis in idiopathic intracranial hypertension after L-P shunt. Neurology 62:514-515, 2004

32. Miller NR: Bilateral visual loss and simultagnosia after lumboperitoneal shunt for pseudotumor cerebri. J Neuroophthalmol 17:36-38, 1997

33. Ogungbo B, Roy D, Gholkar A, Mendelow AD: Endovascular stenting of the transverse sinus in a patient presenting with benign intracranial hypertension. Br J Neurosurg 17:565-568, 2003

34. Owler BK, Parker G, Halmagyi GM, Dunne VG, Grinnell V, McDowell D, et al: Pseudotumor cerebri syndrome: venous sinus obstruction and its treatment with stent placement. J Neurosurg 98:1045-1055, 2003

35. Radhakrishnan K, Ahlskog JE, Cross SA, Kurland LT, O'Fallon WM: Idiopathic intracranial hypertension (pseudotumor cerebri). Descriptive epidemiology in Rochester, Minn, 1976 to 1990. Arch Neurol 50:78-80 1993

36. Rajpal S, Niemann DB, Turk AS: Transverse venous sinus stent placement as treatment for benign intracranial hypertension in a young male: case report and review of the literature. $\mathbf{J}$ Neurosurg 102 (3 Suppl):342-346, 2005

37. Sergott RC, Savino PJ, Bosley TM: Modified optic nerve sheath decompression provides long-term visual improvement for pseudotumor cerebri. Arch Ophthalmol 106:1384-1390, 1988

38. Spoor TC, McHenry JG: Long-term effectiveness of optic nerve sheath decompression for pseudotumor cerebri. Arch Ophthalmol 111:632-635, 1993

39. Spoor TC, Ramocki JM, Madion MP, Wilkinson MJ: Treatment of pseudotumor cerebri by primary and secondary optic nerve sheath decompression. Am J Ophthalmol 112:177-185, 1991

40. Sugarman HJ, Felton WL III, Salvant JB Jr, Sismanis A, Kellum JM: Effects of surgically induced weight loss on idiopathic intracranial hypertension in morbid obesity. Neurology 45:16551659,1995

41. Tomsak RL, Niffenegger AS, Remler BF: Treatment of pseudotumor cerebri with Diamox (acetazolamide). J Clin Neuroophthalmol 18:93-98, 1988

42. Tsai JC, Petrovich MS, Sadun AA: Histopathological and ultrastructural examination of optic nerve sheath decompression. Br J Ophthalmol 79:182-185, 1995

Manuscript submitted August 27, 2007.

Accepted September 5, 2007.

This study was supported by an unrestricted grant to the University of Rochester, Department of Ophthalmology (Dr. Feldon, principal investigator) from Research to Prevent Blindness.

Address correspondence to: Steven E. Feldon, M.D., 210 Crittenden, Box 659, Rochester, New York 14642. email: Steven Feldon@urmc.rochester.edu. 